\title{
Influência de um cultivo de camarão sobre o metabolismo bêntico e a qualidade da água
}

\author{
Ubiratan de Freitas' ${ }^{1}$, Luis F. H. Niencheski ${ }^{1}$, Simoni Zarzur ${ }^{1}$, Rogério P. Manzolli', João P. P. Vieira ${ }^{1}$ \& Leonardo C. Rosa ${ }^{2}$
}

\begin{abstract}
RESUMO
Atividades de cultivo de camarão tendem a aumentar a taxa de sedimentação de matéria orgânica nos corpos hídricos receptores de seus efluentes, devido à excessiva produção de produtos de excreção e sobras de ração. Dependendo das condições ambientais, o sistema pode não apresentar condições de autodepuração, gerando um grande acúmulo de nutrientes ao longo da camada sedimentar e intensificação do metabolismo bêntico levando, muitas vezes, o ambiente a um processo de eutrofização e possível situação de anoxia. Utilizaram-se, no presente estudo, incubações in situ com câmaras bentônicas transparentes e opacas, para avaliar o metabolismo bêntico em uma área de cultivo de camarão no estuário da Lagoa dos Patos, RS. Ficou evidente a existência de uma clara interferência do cultivo nos processos de regeneração bêntica de nutrientes, incrementando os fluxos de nitrogênio (na forma de amônio) e fósforo (fosfato). Embora a interferência desta atividade se tenha mostrado de abrangência local e temporária (apenas 4 a 5 meses do ano), é conveniente salientar que ela ganha maior importância em locais rasos e de circulação restrita, como é o caso de baías e enseadas, locais em que o cultivo de camarão é desenvolvido no estuário da Lagoa dos Patos.
\end{abstract}

Palavras-chave: carcinicultura, câmaras bentônicas, mineralização, fluxos de nutrientes

\section{Influence of shrimp cultivation on the benthic metabolism and water quality}

\begin{abstract}
Shrimp farming activities tend to increase the organic matter sedimentation rate, by extreme inputs of excreta products and shrimp food (commercial shrimp diet) into the environment. In certain environmental conditions, the auto-depuration system can be surpassed, generating a high production of nutrients at the sedimentary layer as a result of benthic metabolism intensification. Consequently the system can be induced to a eutrophication processes and anoxia. In the present study, in situ incubations with transparent and opaque chambers have been used to evaluate the benthic metabolism in semi-intensive shrimp farming areas in the Patos Lagoon Estuary (pen enclosures). Existence of clear interference of the cultivation in the processes of benthic regeneration of nutrients was evident, resulting in increases of phosphorus (phosphate) and nitrogen (in the ammonium form) fluxes. Even considering that this activity has a local and temporary distribution (only 4 to 5 months of the year), it is important to call attention to the sediment oxygen consumption and the input of nutrients deriving from the regeneration of organic matter, which is enhanced in shallow waters and restricted circulation areas (such as small bays), locations where shrimp cultivation is developed in the Patos Lagoon Estuary.
\end{abstract}

Keywords: shrimp farming, benthic chambers, mineralization, nutrients flux

Laboratório de Hidroquímica/FURG. Av. Itália Km 8, CP 474, CEP 96201-900, Rio Grande, RS. Fone: (53) 3233-6516, 3233-6540. E-mail: oceuf@yahoo.com.br; dqmhidro@furg.br; simonizarzur@yahoo.com.br; rogeriomanzolli@bol.com.br; joaopedro_vieira@yahoo.com.br

${ }^{2}$ Laboratório de Ecologia de Invertebrados Bentônicos/FURG. Fone: (53) 3233-6533. E-mail: cielcr@furg.br 


\section{INTRODUÇÃO}

O cultivo de camarão é uma das atividades econômicas que mais crescem em vários países do mundo. No Brasil, a associação do clima favorável e o domínio das novas tecnologias de produção, colocam o País como um dos principais produtores de camarão das Américas (Poersch, 2004). Este setor do agronegócio é relativamente novo mas já apresenta grande expansão, cujo crescimento registrado na última década foi de $20 \%$ ao ano (Lacerda et al., 2006); entretanto, apesar das vantagens econômicas e de tradicionalmente ser considerada uma das atividades do setor primário de baixo impacto ambiental, sérios danos ambientais têm sido reportados em decorrência desta atividade (Alonso-Rodrígues \& Páez-Ozuna, 2003). As águas oriundas dos cultivos têm alta concentração de material orgânico em suspensão e nutrientes, especialmente nitrogênio e fósforo (Jones et al., 2001; Burford et al., 2003), resultado, basicamente, dos restos de alimento fornecido aos camarões (ração), excreção, fitoplâncton e fertilizantes, que geram um potencial para a eutrofização das águas costeiras (Jackson et al., 2004).

$\mathrm{O}$ intenso aporte de MO particulada resulta no aumento das taxas de sedimentação, promovendo uma colonização bacteriana maior (Aller \& Yingst, 1980), intensificando a mineralização desta matéria orgânica (MO) e a regeneração bêntica de nutrientes (Canfield, 1989). Segundo Hargreaves (1998), esta MO é altamente lábil, com baixa relação C:N, que favorece a rápida decomposição e o aumento dos fluxos de nutrientes na interface sedimento-coluna d’água.

A escolha de locais impróprios para o desenvolvimento desta atividade, associada a um manejo inadequado agrava seu potencial poluidor já que o aporte excessivo de MO em locais de baixa hidrodinâmica pode ultrapassar sua capacidade de mineralização, que tenderá a se acumular no sedimento (Boyd, 2001; Trott et al., 2004). A intensificação dos processos de decomposição causada pelo acúmulo de MO no sedimento, frequentemente rege a qualidade de água nesses ambientes, resultando em uma depleção de oxigênio na coluna d’água e tornando os sedimentos anóxicos. As vias anaeróbicas de decomposição são favorecidas aumentando, assim, a produção e liberação na coluna d'água de compostos tóxicos reduzidos, como amônia, sulfetos (Alongi et al., 1999), manganês (na forma $\mathrm{Mn}^{+2}$ ), metano (Avnimelech \& Ritvo, 2003) e nitrito (Jiménez-Montealegre et al., 2002); como conseqüência dessa cadeia de processos, além do potencial de eutrofização já mencionado, a estrutura da comunidade bentônica pode ser fortemente afetada (Jackson et al., 2004). Problemas como os elencados acima, associados à falta de conhecimento dos processos biogeoquímicos, têm causado intensa preocupação em muitos países onde são desenvolvidas atividades aquícolas (Hinrichorasen, 1998).

Segundo Warnken et al. (2002), o sedimento desempenha papel crucial na ciclagem de nutrientes e pode funcionar como a principal fonte de nutrientes para a coluna d'água, sobretudo em ecossistemas costeiros de baixa profundidade, como é o caso do estuário da Lagoa dos Patos. A compreensão dos processos acima citados ajudaria na predição de impactos e adoção de práticas nesta atividade para o uso sustentável do ambiente marinho. Estudos biogeoquímicos, conduzidos em ambientes sob efeito de cultivos de camarão, são escassos. Os trabalhos de regeneração bêntica de nutrientes nesses locais são ainda bastante recentes e se restringem a poucos experimentos, conduzidos especialmente na Austrália. Medidas de fluxos de nutrientes permitem inferir sobre as principais vias do processo de mineralização e determinar, em caráter quantitativo, a importância do sedimento no ciclo global de nutrientes em viveiros de cultivos (Riise \& Roos, 1997).

No Rio Grande do Sul, apesar da carcinicultura comercial, utilizando o camarão exótico Litopenaeus vannamei, ser bastante incipiente, está em pleno crescimento e já se mostra como atividade do ramo do agronegócio, muito promissora e atraente aos novos investidores. Paralelo aos cultivos comerciais, a Fundação Universidade Federal do Rio Grande (FURG) vem, através do Laboratório de Maricultura (EMA), incentivando e desenvolvendo, junto aos pescadores artesanais, a nível de pesquisa, o cultivo do camarão-rosa Farfantepenaeus paulensis (espécie nativa) em cercados instalados em uma enseada rasa, denominada Saco do Justino.

Este rápido crescimento da atividade na região deve ser avaliado com cautela e, portanto, estudos prévios de potenciais impactos ao meio ambiente são necessários em sua fase inicial, para que esta região e o Brasil não passem por experiências desoladoras como as acontecidas no Equador, China, Indonésia e Tailândia.

Objetivou-se, então, com o presente trabalho, avaliar os efeitos causados por um cultivo de camarão (desenvolvido em cercados) sobre o metabolismo bêntico e a qualidade da água. Utilizaram-se, no experimento, incubações in situ com câmaras bentônicas para medição dos fluxos de nutrientes na interface sedimento-água e se avaliou também a estrutura da comunidade bentônica.

Ressalta-se que este estudo é inédito no Brasil, onde a atividade de carcinicultura vem crescendo rapidamente e pouco de seus impactos tem sido avaliado.

\section{MATERIAL E MÉTODOS}

\section{Local e características do cultivo}

O experimento foi realizado na enseada do Saco do Justino, localizada na porção sul do estuário da Lagoa dos Patos ( $32^{\circ} 3^{\prime} 55^{\prime}$ ' S, 52 $12^{\circ}$ ' 30” W), na cidade de Rio Grande, RS (Figura 1).

Localizada na planície costeira do Rio Grande do Sul, com $10.360 \mathrm{~km}^{2}$, a Lagoa dos Patos recebe águas da extensa bacia hidrográfica, compreendida pelo complexo lagunar $\mathrm{Pa}-$ tos-Mirim que, juntas, constituem o maior complexo lagunar costeiro da América do Sul (Herz, 1977).

A área estuarina representa cerca de $10 \%$ da área total da Lagoa dos Patos. Nas áreas de canal possui uma profundidade média de $6 \mathrm{~m}$ mas aproximadamente $80 \%$ da região estuarina tem menos de $2 \mathrm{~m}$ de profundidade. As áreas de menor profundidade $(\approx 0,5 \mathrm{~m})$ são representadas por enseadas que, regionalmente, são denominadas sacos e as áreas 


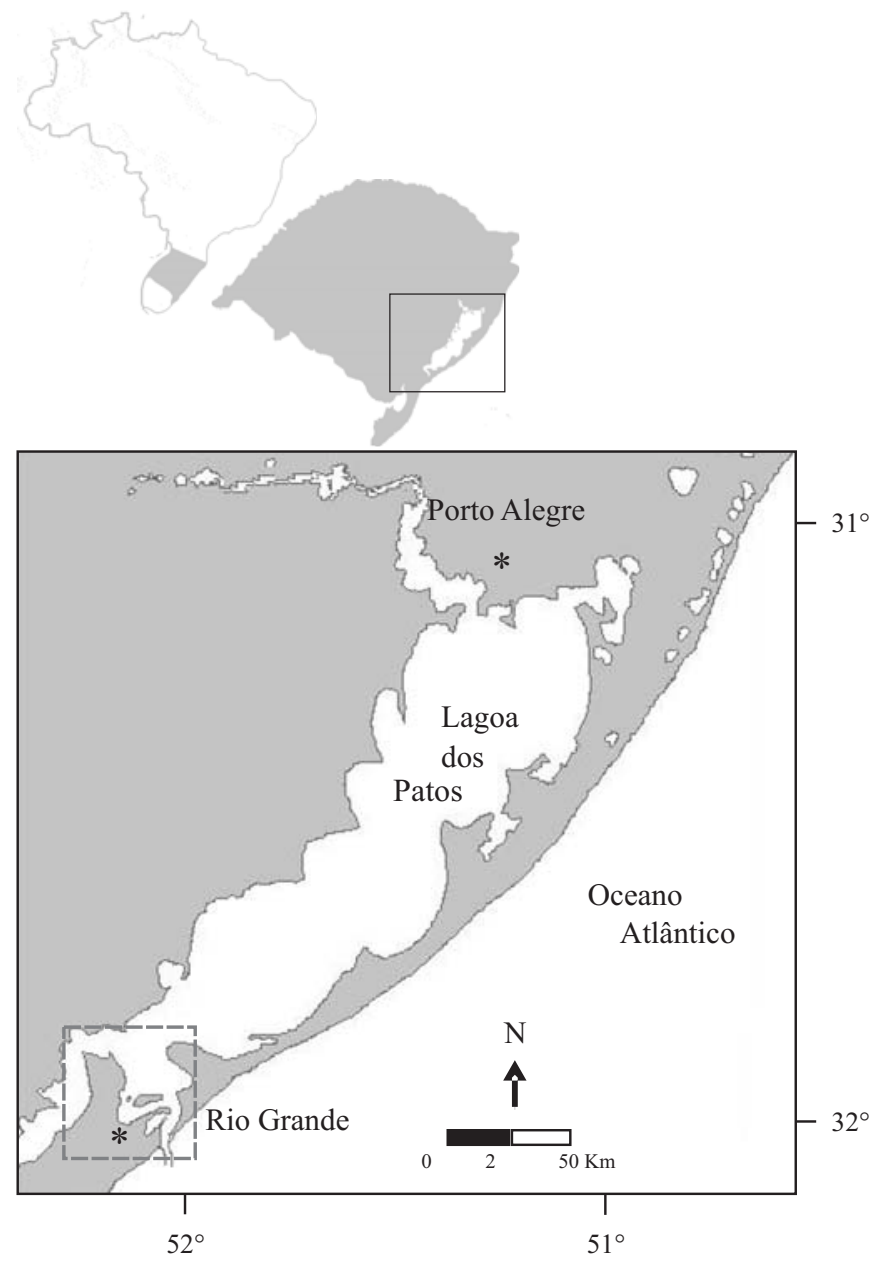

Figura 1. Localização da área de estudo

de maior profundidade $(\approx 2-15 \mathrm{~m})$ correspondem aos canais de navegação primários e secundários (Castello, 1985).

Como típico de lagoas “estranguladas”, o canal da Lagoa dos Patos, com largura de $800 \mathrm{~m}$, atenua os avanços das ondas de maré $(0,47 \mathrm{~m})$ para dentro do estuário. Os ventos do quadrante sul, predominantemente o SW, favorecem a penetração de água salgada pela Barra de Rio Grande (enchente) (Fernandes \& Niencheski, 1998). A salinidade varia de 0 a 35 e a temperatura apresenta um claro padrão sazonal, com valores máximos $\left(27^{\circ} \mathrm{C}\right)$, durante o verão e mínimos $\left(10^{\circ} \mathrm{C}\right)$, durante o inverno (Kantin \& Baumgarten, 1982).

A profundidade do local de cultivo dos camarões se manteve próxima a $0,6 \mathrm{~m}$, sendo o sedimento composto, predominantemente, de areia fina, silte e argila. O cultivo teve duração total de 86 dias, entre os meses de janeiro e abril de 2004. O cercado, com $50 \mathrm{~m}^{2}$ de área, se compunha de rede de poliéster revestida de PVC, com abertura de malha de $5,0 \mathrm{~mm}$ e altura de 2,1 $\mathrm{m}$, sendo sustentado por bambus. A densidade de estocagem foi de 20 camarões $\mathrm{m}^{-2}$, habitualmente empregada no cultivo desta espécie no estuário da Lagoa dos Patos (Cavalli et al., 2003). Usou-se, para a alimentação dos camarões, ração comercial peletizada, sendo que, a partir da terceira semana, os camarões foram alimentados somente com ração de engorda (35\% de proteína bruta) em alimentadores tipo bandeja. O cultivo foi mantido ao longo de todo o experimento (desde a larvicultura até a despesca) por alunos do Programa de Pós-Graduação em Aqüicultura da FURG.

\section{Amostragem com câmaras bentônicas e análises físico- químicas}

Duas campanhas amostrais foram realizadas, em que: a Campanha 1 ocorreu no final de janeiro (antes do povoamento com as pós-larvas) e a Campanha 2 em meados de abril (3 dias antes da despesca), exatamente no mesmo local da Campanha 1; desta forma, a primeira serviu de referência (controle) para comparação com a segunda, após um ciclo completo de cultivo. Ressalta-se que a técnica empregada envolveu medições diretas por incubação in situ, com câmaras bentônicas transparentes e opacas, segundo metodologia descrita em Zarzur (2001), visando quantificar o metabolismo bêntico, em função do consumo de oxigênio pelo sedimento e dos fluxos de regeneração bêntica dos nutrientes inorgânicos dissolvidos (nitrogênio amoniacal total, nitrito, nitrato, fosfato e silicato). Utilizaram-se, em todas as incubações, três réplicas de cada câmara (3 transparentes e 3 opacas), objetivando minimizar os efeitos da heterogeneidade espacial. Cada câmara é composta de um domo de acrílico (semi-esfera) de $40 \mathrm{~cm}$ de diâmetro e confeccionada em acrílico cristal (câmaras transparentes) e preto (câmaras opacas) (Figura 2). As câmaras isolam um volume de aproximadamente 16,7 L sobre uma área de sedimento de $0,125 \mathrm{~m}^{2}$ e dispõem de um agitador manual, visando promover a homogeneização e evitar a formação de gradientes de concentração dentro da câmara.

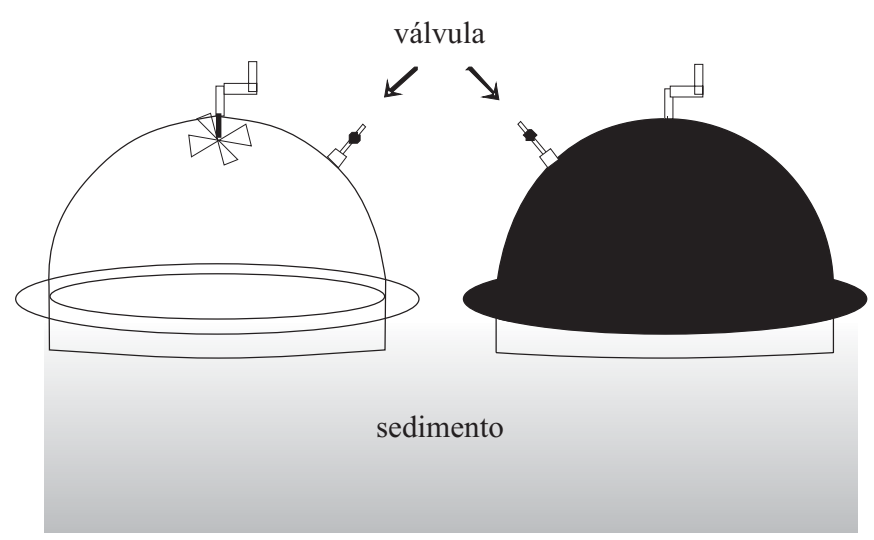

Figura 2. Desenho esquemático das câmaras transparente e opaca fixadas ao sedimento, destacando-se a válvula de tomada de água. Observa-se também a hélice de homogeneização interna

As câmaras foram fixadas ao sedimento, manualmente, e as incubações tiveram duração de $24 \mathrm{~h}$. A coleta das amostras de água de seu interior foi feita utilizando-se seringas plásticas de $60 \mathrm{~mL}$ através de uma válvula, com controle de fechamento e abertura, enquanto a água incubada no interior das câmaras foi homogeneizada a cada 2 h e tomadas alíquotas a cada 6 h, para determinação dos nutrientes inorgânicos dissolvidos (nitrogênio amoniacal total, nitrito, nitrato, fosfato e silicato), oxigênio dissolvido, $\mathrm{pH}$, temperatura, 
salinidade e clorofila- $a$. O nitrogênio amoniacal total, também chamado amônio total, compreende a soma da forma iônica (amônio $-\mathrm{NH}_{4}^{+}$) e gasosa (amônia $-\mathrm{NH}_{3}$ ) e se usará apenas a designação amônio para fazer referência ao nitrogênio amoniacal $\left(\mathrm{NH}_{3}+\mathrm{NH}_{4}{ }^{+}\right)$.

$\mathrm{O}$ gradiente de concentração dos nutrientes determinados ao longo de 24 h de incubação representou as trocas entre a interface sedimento-coluna d'água, dando origem a um valor de fluxo, calculado segundo Hargrave \& Connoly (1978) e modificado por Freitas (2006). A transferência dos componentes dissolvidos do sedimento para a coluna d'água resulta em um aumento de concentração conferindo valores positivos para os fluxos (liberação ou efluxo) e, em situação inversa, valores negativos (consumo ou influxo).

Da água amostrada mediu-se imediatamente a temperatura, $\mathrm{pH}$, salinidade e oxigênio dissolvido; para análise dos nutrientes a amostra foi filtrada logo após a coleta, em filtro de acetato de celulose de 0,45 $\mu \mathrm{m}$; para a análise de nitrito, nitrato, fosfato e silicato, as amostras, já filtradas, foram acondicionadas em frascos de polietileno e guardadas em freezer para posterior análise; contudo, adicionaram-se os reagentes específicos às amostras destinadas a análise do amônio, antes de serem congeladas.

\section{Parâmetros considerados e métodos analíticos}

A temperatura foi medida por termômetro de mercúrio; a salinidade, através de um salinômetro de indução, modelo “Yellow Spring 33 SCT”; o pH, por um pHmetro digital $(0,01$ de precisão), modelo Digimed DM2; o oxigênio dissolvido foi determinado segundo Strickland \& Parsons (1972), com base no método iodométrico clássico de Winkler, adaptado para microvolumes; nitrito $\left(\mathrm{N}-\mathrm{NO}_{2}{ }^{-}\right)$, nitrato $\left(\mathrm{N}-\mathrm{NO}_{3}{ }^{-}\right)$, fosfato $\left(\mathrm{P}-\mathrm{PO}_{4}{ }^{3-}\right)$ e silicato $\left(\mathrm{SiO}_{4}{ }^{4-}\right)$ foram definidos através de métodos absorciométricos, descritos por Aminot \& Chaussepied (1983) e adaptados por Baumgarten et al. (1996); determinouse o amônio $\left(\mathrm{NH}_{3}+\mathrm{NH}_{4}^{+}\right)$pelo método absorciométrico, descrito por Solorzano (1969) e adaptado por Baumgarten et al. (1996); a razão N/P foi estimada pela razão molar entre o somatório das concentrações dos nitrogenados dissolvidos (amônio, nitrito e nitrato) e fosfato; mediu-se, enfim, a Cl-a através do método fluorimétrico (Fluorímetro modelo TD-700).

\section{Macroinvertebrados bentônicos}

Ao final das incubações sob cada câmara foi introduzido, no sedimento, um tubo de PVC de $10 \mathrm{~cm}$ de diâmetro e retirados os 20 cm superficiais; o sedimento foi, então, peneirado em uma rede de malha $300 \mu \mathrm{m}$ utilizando-se água do próprio ambiente e o material retido foi fixado com formol a $10 \%$.

No Laboratório de Ecologia de Invertebrados Bentônicos da FURG, os organismos foram identificados ao menor nível taxonômico possível e quantificados.

\section{RESULTADOS E DISCUSSÃO}

\section{Caracterização da coluna da água}

Antes do cultivo predominaram águas mais quentes e menos salinas (Tabela 1). Em ambas as campanhas, o pH esteve próximo a 7,5 e o OD acima de $8 \mathrm{mg} \mathrm{L}^{-1}$. As águas se encontravam super-satuaradas na Campanha 1 (116\%), provavelmente em função da grande presença de macrófitas observadas no fundo da enseada antes da instalação do cercado, somado à ação dos ventos; neste período a biomassa fitoplanctônica foi metade daquela encontrada no final do cultivo, ao contrário dos valores de amônio e nitrito, que dobraram suas concentrações ao fim do cultivo; entretanto, os teores de nitrito são praticamente desprezíveis. Na campanha 2 a concentração de amônio foi de $0,2 \mathrm{mg} \mathrm{L}^{-1}$ e, como conseqüência, a relação N/P foi consideravelmente alta, em torno de 39.

Tabela 1. Parâmetros físico-químicos analisados no cultivo em cercados (média das alíquotas tomadas na primeira amostragem - às $10 \mathrm{~h}$ - do interior das seis câmaras

\begin{tabular}{lcc}
\hline \multirow{2}{*}{ Parâmetros } & \multicolumn{2}{c}{ Campanha } \\
\cline { 2 - 3 } Temperatura $\left.{ }^{\circ} \mathrm{C}\right)$ & $\mathbf{1}$ & $\mathbf{2}$ \\
Salinidade & 4 & 22 \\
$\mathrm{pH}$ & 7,38 & 15 \\
OD $\left(\mathrm{mg} \mathrm{L}^{-1}\right)$ & 8,9 & 7,5 \\
$\%$ Saturação OD & 116,1 & 8,1 \\
Amônio $\left(\mathrm{mg} \mathrm{L}^{-1}\right)$ & 0,098 & 99,9 \\
Nitrito $\left(\mathrm{mg} \mathrm{L}^{-1}\right)$ & 0,0005 & 0,202 \\
Nitrato $\left(\mathrm{mg} \mathrm{L}^{-1}\right)$ & 0,0238 & 0,0012 \\
Fosfato $\left(\mathrm{mg} \mathrm{L}^{-1}\right)$ & 0,015 & 0,0269 \\
Razão N/P (razão molar) & 18 & 0,013 \\
Silicato $\left(\mathrm{mg} \mathrm{L}^{-1}\right)$ & 2,96 & 39 \\
Cl-a $\left.(\mu \mathrm{g} \mathrm{L})^{-1}\right)$ & 4,2 & 0,65 \\
\hline
\end{tabular}

Os valores de fosfato parecem não ter sofrido alteração significativa, sendo ligeiramente mais elevados no início (0,015 mg L $\left.{ }^{-1}\right)$. O silicato exibiu maiores concentrações no primeiro experimento em função da contribuição dos aportes continentais de água doce, que resultaram na queda da salinidade $(S=4)$.

\section{Fluxos dos elementos dissolvidos na interface sedimento- coluna da água \\ Oxigênio: O gradiente de oxigênio formado ao longo das} $24 \mathrm{~h}$ de incubação e determinado pelo balanço total do $\mathrm{O}_{2}$ (produção e consumo), deu origem a um fluxo ao qual se denomina Consumo de Oxigênio pelo Sedimento (COS), com valores negativos, quando o consumo supera a produção e vice-versa; nas câmaras opacas, como não há produção, o COS representa simplesmente a demanda por oxigênio que determinada área de sedimento possui, medida por período de tempo (Figura 3).

Visto que os microrganismos heterotróficos utilizam o $\mathrm{O}_{2}$ como receptor final de elétrons para a degradação aeróbica da matéria orgânica, o COS é um importante índice amplamente utilizado para estimar as taxas de regeneração bêntica (Thamdrup \& Canfield, 2000). Ressalta-se que, no presente estudo, não se determinou a produção primária pelágica; a qual poderia ser descontado do COS obtido nas incubações com as câmaras bentônicas e se obter, então, o valor líquido da produção primária bentônica. Assim, é 
A.

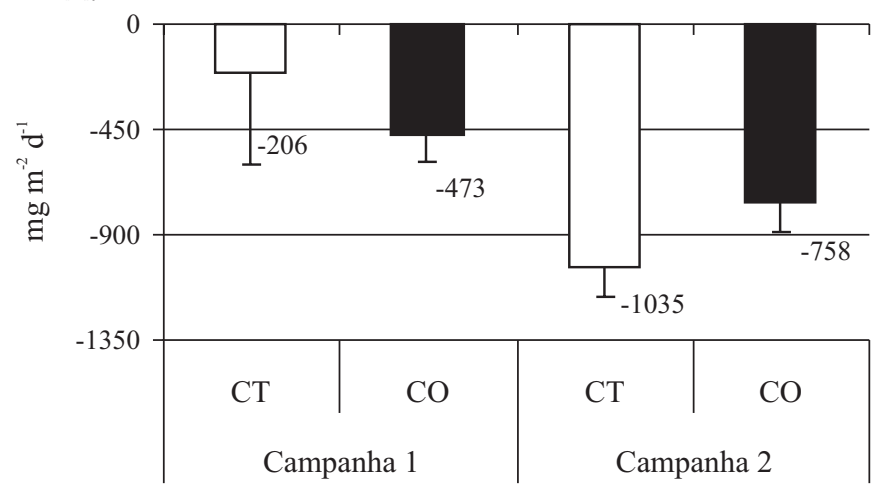

C.

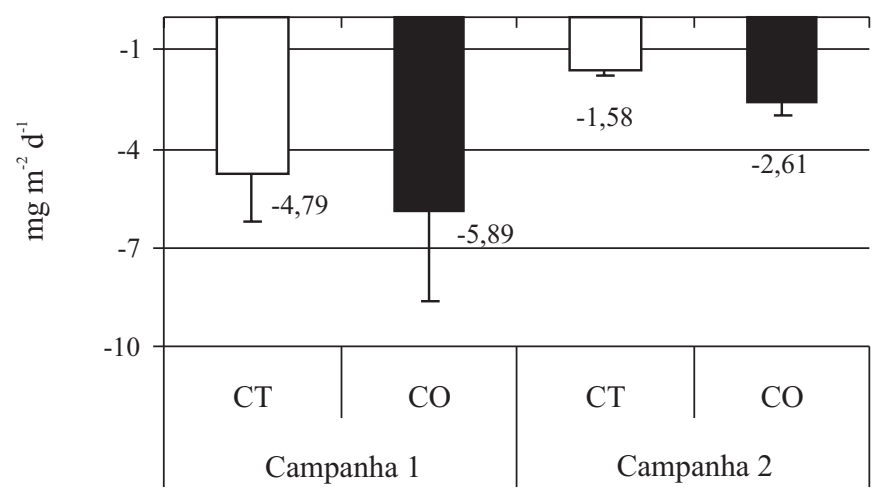

E.

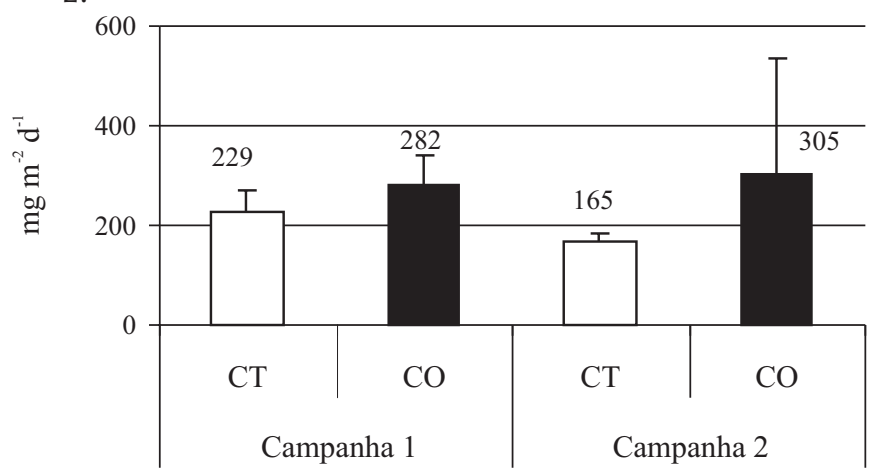

B.

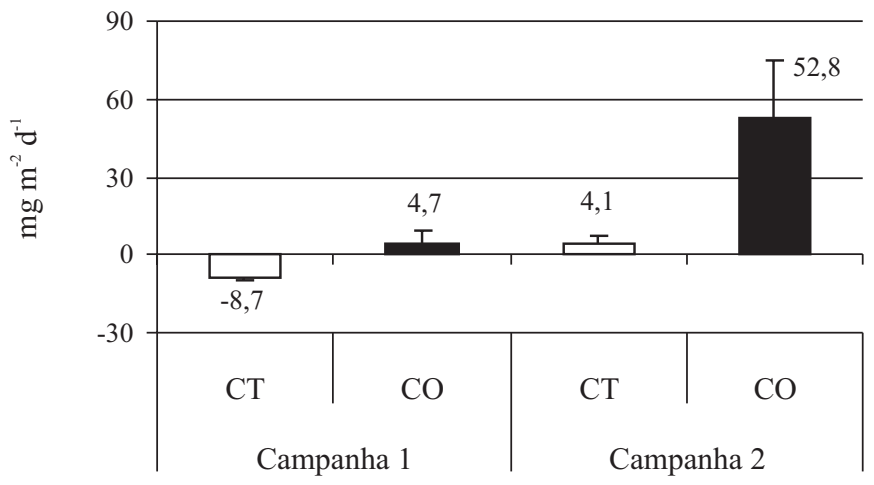

D.

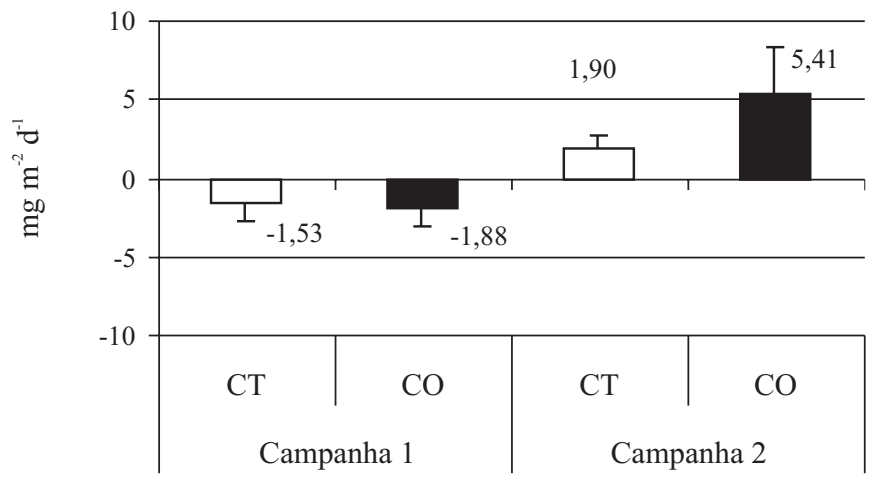

F.

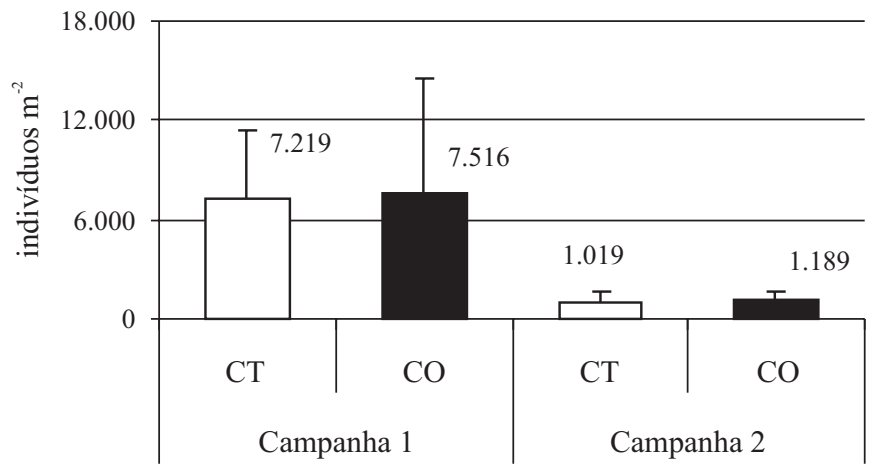

Figura 3. Consumo do oxigênio pelo sedimento (A), fluxos de amônio (B), nitrato (C), fosfato (D) e silicato (E), expressos em $\mathrm{mg} \mathrm{m}^{-2} \mathrm{~d}^{-1}$ e densidade de macroinvertebrados bentônicos $(\mathrm{F})$, expressa em indivíduos $\mathrm{m}^{-2}$

possível que tenha havido uma ligeira superestimação do COS nas câmaras opacas.

Antes do cultivo o COS médio foi de -206 e -473,4 $\mathrm{mgO}_{2}$ $\mathrm{m}^{-2} \mathrm{~d}^{-1}$, nas CT e CO, respectivamente. O COS nas CT é menor em função da produção primária que repõe parte do oxigênio consumido na mineralização. Foi notável o incremento do COS ao final do período de cultivo, quando se registraram valores de $-1034,9$ e $-758 \mathrm{mgO}_{2} \mathrm{~m}^{-2} \mathrm{~d}^{-1}$, nas CT e $\mathrm{CO}$, respectivamente; aparentemente, o valor mais elevado registrado nas CT leva a se pensar que ocorreu um equívoco, pois se o fitoplâncton injeta $\mathrm{O}_{2}$ no interior das CT durante as horas do dia, como poderia o COS ser superior ao das $\mathrm{CO}$, onde há somente consumo?

Aquele valor nada mais é do que o reflexo da alta produção de $\mathrm{O}_{2}$ ocorrida durante o dia, seguida de um alto con- sumo à noite resultando, por fim, em elevado gradiente de concentração ao longo do tempo de incubação. Hickey (1985) cita que a atividade fotossintética durante o dia leva a um incremento do COS. Segundo Fenchel \& Glud (2004), na presença de luz a atividade autotrófica resulta na liberação de exudados de carbono altamente lábil (matéria orgânica dissolvida), estimulando a atividade heterotrófica microbiana o que resulta em intensas taxas de consumo de oxigênio à noite.

Ao se considerar o COS da CT (coleta 2) de -1034,9 mgO $_{2}$ $\mathrm{m}^{-2} \mathrm{~d}^{-1}$, uma profundidade em torno de $0,6 \mathrm{~m}$ e uma concentração média diária de OD de $8 \mathrm{mg} \mathrm{L}^{-1}$, o metabolismo bêntico representará cerca de $21,5 \%$ do estoque de OD da coluna d’água.

Nazário et al. (2005) reportam valores médios de COS sob 
cultivos de ostra na Baía de Guaratuba, PR, de -0,41 mgO $\mathrm{m}^{-2} \mathrm{~d}^{-1}$ (câmaras opacas) e Machado (1989) reporta valores em torno de $-2.000 \mathrm{mgO}_{2} \mathrm{~m}^{-2} \mathrm{~d}^{-1}$ para a lagoa Guarapina, no Rio de Janeiro.

A amplitude dos valores de fluxos reportados mundialmente é muito grande (Forja et al., 2004), uma vez que variam enormemente em função das características ambientais, intrínsecas de cada local e em função da metodologia utilizada para a medição dos fluxos. São raros os estudos de metabolismo e regeneração bêntica em áreas de cultivo de camarão e praticamente inexistentes aqueles executados com câmaras bentônicas. A grande maioria se utiliza de métodos indiretos ou de laboratório, tais como fluxos teóricos de difusão molecular, de modelagens ou através de incubações in vitro, razão pela qual é importante se ter pontos ou valores de referência obtidos na própria região para comparação dos resultados. Para se ter uma idéia, da mesma forma que são relatados valores bastante inferiores aos encontrados no presente estudo (em áreas de cultivos), também se acham valores muito superiores em ambientes costeiros não impactados. Segundo Alongi et al. (1999), grandes variações de fluxos são encontradas até mesmo em escalas de poucos metros.

De qualquer forma, ficou evidente, no presente estudo, o aumento do metabolismo bêntico ao final do cultivo, mostrando que houve nítida interferência desta atividade; entretanto, a influência deve ficar restrita, provavelmente, ao cercados e seu entorno próximo, visto que a própria estrutura (malha) dos cercados forma um obstáculo à dispersão do material orgânico particulado, favorecendo sua sedimentação, em grande parte, no interior do sistema de cultivo.

Nitrogênio inorgânico dissolvido (NID: amônio, nitrito e nitrato): Em média, os maiores fluxos de liberação de NID foram registrados na forma de amônio, tanto antes como depois da instalação dos cultivos (Figura 3). Durante a primeira campanha foi notório o consumo nas CT e liberação nas CO $\left(-8,7\right.$ e $4,7 \mathrm{mg} \mathrm{m}^{-2} \mathrm{~d}^{-1}$, respectivamente). Os fluxos de nitrito foram praticamente desprezíveis (0,04 e -0,1 $\mathrm{mg} \mathrm{m}^{-2} \mathrm{~d}^{-1}$, nas CT e CO, nesta ordem); os fluxos de nitrato foram negativos em ambas as câmaras, com valores médios de $-4,5 \mathrm{mg} \mathrm{m}^{-2} \mathrm{~d}^{-1}$ nas CT e de $-5,9 \mathrm{mg} \mathrm{m}^{-2} \mathrm{~d}^{-1}$ nas CO; já os valores negativos de amônio e nitrato nas câmaras transparentes estão relacionados à maior assimilação desses constituintes, por parte dos produtores primários. Observa-se, nas CO, maior consumo de nitrato acompanhado pela liberação de amônio, indicando a ocorrência de processos de denitrificação em que, sob condições de baixa concentração de OD e disponibilidade de nitrato, este elemento pode ser utilizado por determinados microrganismos, como o aceptor final de elétrons (respiração anaeróbica), sendo, então, convertido a amônio $\left(\mathrm{NO}_{3}^{-}\right.$à $\left.\mathrm{NH}_{4}{ }^{+}\right)$.

Ao contrário da denitrificação, a nitrificação transforma o amônio em nitrato $\left(\mathrm{NH}_{4}^{+}\right.$à $\left.\mathrm{NO}_{3}{ }^{-}\right)$, razão por que é considerada, em ambientes oxidantes, um dos processos de maior importância no ciclo global do nitrogênio, inclusive em ambientes marinhos, haja vista estar relacionada à presença das diferentes formas do nitrogênio inorgânico dissolvido
$\left(\mathrm{NH}_{4}{ }^{+}, \mathrm{NO}_{2}{ }^{-}\right.$e $\left.\mathrm{NO}_{3}{ }^{-}\right)$, incluindo a perda de $\mathrm{N}$ para a atmosfera (formação de $\mathrm{N}_{2(\mathrm{~g})}$ via denitrificação quando o ambiente se torna redutor). As bactérias nitrificantes também podem competir com os produtores primários pelo íon amônio (Ward, 2000) e, se em excesso, esses microorganismos podem levar ambientes aquáticos à anoxia, como observado em vários estuários (Balls et al., 1996), devido às reações de oxidações do $\mathrm{NH}_{4}^{+}$e do $\mathrm{NO}_{2}^{-}$.

Ao final do cultivo se reduziram os fluxos de nitrato e se intensificaram os de amônio, em especial nas CO (52,8 $\left.\mathrm{mg} \mathrm{m}^{-2} \mathrm{~d}^{-1}\right)$, provavelmente em função de um acúmulo de MO associado a uma condição hipóxica, em que um fluxo dessa magnitude representa um aporte de $\mathrm{N}$ na forma de amônio oriundo da regeneração bêntica para a coluna d’água, de aproximadamente $0,52 \mathrm{Kg} \mathrm{ha}^{-1} \mathrm{~d}^{-1}$, ou seja, 3,6 Kg ha-1 semana-1. O valor médio de amônio na água foi de $0,20 \mathrm{mg} \mathrm{L}^{-1}$; o que equivale a $200 \mathrm{mg} \mathrm{m}^{-3}$ caso se considere uma área de $1 \mathrm{~m}^{2}$ e profundidade de $1 \mathrm{~m}$.

Como o local de cultivo no Saco do Justino apresentou profundidade média de $0,6 \mathrm{~m}$, pode-se dizer que se tem aproximadamente $120 \mathrm{mg} \mathrm{m}^{-2}$ (N-amônio). Um fluxo de $52,8 \mathrm{mg} \mathrm{m}^{-2} \mathrm{~d}^{-1}$ representa cerca de $44 \%$ do teor de amônio na água, o que é um aporte considerável tendo em vista ser o Saco do Justino uma enseada rasa e semi-fechada, cuja circulação é restrita e, portanto, a dispersão dos poluentes é baixa.

Fosfato: Antes de se iniciarem as atividades de cultivo, os fluxos de fosfato foram negativos, mostrando que há remoção deste nutriente da coluna d’água, pelo compartimento bêntico (Figura 3). Os valores registrados nas CT e CO foram de $-1,53 \mathrm{mg} \mathrm{m}^{-2} \mathrm{~d}^{-1} \mathrm{e}-1,88 \mathrm{mg} \mathrm{m}^{-2} \mathrm{~d}^{-1}$, respectivamente.

Ao final do cultivo, os fluxos foram positivos em ambas as câmaras, indicando a liberação deste nutriente do sedimento para a coluna d'água; nas CO o fluxo foi de $5,41 \mathrm{mg} \mathrm{m}^{-2} \mathrm{~d}^{-1} \mathrm{e}$, nas CT, de $1,90 \mathrm{mg} \mathrm{m}^{-2} \mathrm{~d}^{-1}$.

A segunda etapa de amostragem foi dominada por águas mais salinas, que favorecem a desorção do fosfato adsorvido às partículas em suspensão e/ou os ligados aos hidróxidos de ferro, resultando em maior disponibilidade do fosfato dissolvido, que pode ser liberado e se manter biodisponível na coluna d’água (Schenau \& de Lange, 2001); salienta-se, entretanto, que, assim como o nitrogênio, o fósforo também é um constituinte importante das rações de camarão; o acúmulo no sedimento do excedente desta ração tende a elevar as taxas de decomposição promovendo uma regeneração maior do fosfato contribuindo, desta maneira, para os fluxos positivos observados.

Lin \& Nash (1996) estimaram que cerca de $24 \%$ do total de fósforo aplicado na forma de ração em viveiros de cultivo intensivo de camarão, se acumularam nos sedimentos mas esses valores são bastante variáveis e dependem principalmente da forma de manejo do cultivo e do tipo de ração, ou seja, de sua digestibilidade e qualidade.

O fluxo da CO representa um aporte de fósforo para o ambiente, de aproximadamente $0,054 \mathrm{~kg} \mathrm{ha}^{-1} \mathrm{~d}^{-1}$ ou $1,67 \mathrm{~kg} \mathrm{ha}^{-1}$ por mês; considerando-se novamente a profundidade de $0,6 \mathrm{~m}$, tem-se aproximadamente $7,8 \mathrm{mg} \mathrm{m}^{-2}$. Um fluxo de 


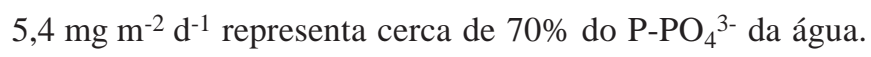
Pereira Filho et al. (1998) estimaram em cerca de 2,5\% este valor sob cultivos de mexilhão.

Esses valores de fluxo do cultivo no Saco do Justino são bastante elevados se comparados com a sua concentração na coluna d’água; um aporte desta magnitude garante, certamente, grande parte do fósforo requerido pelos produtores primários, o que deve ter contribuído para maior biomassa fitoplanctônica, indicada pelos valores de Cl- $a$ mais elevados que o da primeira campanha (de 4,2 para 9,0 $\mu \mathrm{g} \mathrm{L}^{-1}$ ); apesar deste maior aporte de fosfato regenerado, sua concentração na água foi mais baixa que na primeira amostragem $\left(0,013 \mathrm{mg} \mathrm{L}^{-1}\right)$ devido, sem dúvida, à maior assimilação por parte do fitoplâncton, sugerindo que este deva exercer papel fundamental no controle das concentrações de fosfato da coluna d'água.

Silicato: O silicato foi o elemento que apresentou os maiores fluxos em ambas as câmaras e nas duas coletas (Figura 3). Os fluxos foram superiores nas câmaras opacas, evidenciando a absorção pelos produtores primários, provavelmente diatomáceas bentônicas penadas, que são uma porção considerável da biomassa do microfitobêntos no estuário (Abreu, 1992) e necessitam de silício na mesma razão que o nitrogênio (C:Si:N:P = 106:16:16:1, razão molar) (Sigmon \& Cahoon, 1997).

Os fluxos de silicato apresentaram comportamento mais conservativo entre as duas amostragens (fluxos semelhantes), demonstrando não serem influenciados de forma significativa, pela presença dos cultivos; observou-se ainda, que, além dos fluxos mais intensos o silicato exibiu as maiores variações entre as câmaras, com altos valores de desvios-padrão. Sabendo-se que as bactérias exercem particular importância no processo de dissolução das frústulas silicosas de diatomáceas e que, em geral, o microfitobentos é de distribuição heterogênea (Glud et al., 2002), prevê-se maior variação espacial dos fluxos.

De forma geral e se comparando os resultados dos fluxos de silicato com os de $\mathrm{N}$ e $\mathrm{P}$, pode-se afirmar que, de fato, o cultivo teve interferência no metabolismo bêntico, já que não houve incremento considerável nos fluxos deste elemento, como ocorreu para o COS e fluxos de N e P. Diferente destes, o Si não está diretamente relacionado aos aportes oriundos do cultivo, pois se desconhece ser ele um componente considerável da ração ou das fezes dos camarões, corroborando para a hipótese acima.

\section{Macroinvertebrados bentônicos}

A densidade total dos macroinvertebrados bentônicos é mostrada na Figura 3, na qual se identificaram 7 táxons, sendo eles: Heteromastus similis, Nephtys fluviatilis, Laeonereis acuta, Erodona mactroides, Cyrtograpsus angulatus, Nemertino e Chironomidae.

Não houve diferença expressiva entre as CT e CO, seja na primeira campanha (7.519 e 7.516 ind $\mathrm{m}^{-2}$, nas CT e CO, respectivamente) ou na segunda (1.019 e 1.169 ind $\mathrm{m}^{-2}$, nas mesmas câmaras); entretanto, observou-se uma clara diferença temporal sendo que, inicialmente, os organismos bentônicos eram muito mais abundantes que depois da instalação dos cultivos; entre as cinco espécies identificadas, se destacam por sua densidade, em especial na primeira amostragem, os poliquetas Heteromastus similis, Nephtys fluviatilis e Laeonereis acuta, e o pelecípode Erodona mactroides (Tabela 2), todos tipicamente estuarinos e representantes da infauna do estuário da Lagoa dos Patos (Bemvenuti, 1998).

Tabela 2. Número total de taxons de macroinvertebrados bentônicos identificados, suas respectivas densidades (indivíduos. $\mathrm{m}^{-2}$ ) e desvio padrão (DP)

\begin{tabular}{|c|c|c|c|c|}
\hline \multirow{2}{*}{ Taxons } & \multicolumn{2}{|c|}{ Campanha 1} & \multicolumn{2}{|c|}{ Campanha 2} \\
\hline & ind $m^{-2}$ & $\mathrm{DP}$ & ind $\mathrm{m}^{-2}$ & DP \\
\hline Heteromastus similis & 3.476 & 4.371 & 837 & 540 \\
\hline Nephtys fluviatilis & 1.838 & 409 & 55 & 68 \\
\hline Laeonereis acuta & 928 & 978 & 36 & 96 \\
\hline Erodona mactroides & 455 & 428 & 36 & 62 \\
\hline Nemertino & 36 & 96 & 36 & 96 \\
\hline Chironomidae & 18 & 48 & 0 & 0 \\
\hline Cyrtograpsus sp & 18 & 48 & 0 & 0 \\
\hline
\end{tabular}

Apesar de Heteromastus similis ter alcançado altas densidades na primeira amostragem (cerca de 3.476 ind $\mathrm{m}^{-2}$ ), na segunda foi de apenas 837 ind $\mathrm{m}^{-2}$, ou seja, uma diminuição de 76\%; a redução foi ainda maior quando consideradas as outras espécies como, por exemplo, os poliquetas Nephtys fluviatilis e Laeonereis acuta, cuja redução foi maior que 96\%. Segundo Bemvenuti (1994), não é característica de Nephtys fluviatilis se enterrar a maiores profundidades no sedimento; portanto, nas camadas superficiais estaria mais susceptível às interferências inerentes ao cultivo.

Confiorme Bemvenuti (1992), a infauna mantém as densidades elevadas através de mecanismos de escape a predação, como a capacidade de enterramento e a mobilidade dos organismos. A maior persistência do poliqueta Heteromastus similis após a introdução dos cultivos, deve estar relacionada a tal estratégia. Em um estudo sobre a composição de invertebrados bentônicos sob cultivos em cercados na mesma área do presente estudo, Soares et al. (2004) encontraram uma redução da densidade total do bentos de aproximadamente $86 \%$ após 21 dias, passado este período houve, também, notáveis diferenças entre o interior dos cercados e a área controle, atribuídas basicamente à predação e/ou perturbação do sedimento.

A panagem dos cercados, associada à diminuição da velocidade de corrente pela presença dos cercados, pode ser fator que contribua para a diminuição do recrutamento de larvas de invertebrados bentônicos, resultando na queda da densidade e riqueza de espécies observadas.

Embora não se tenha efetuado a caracterização sedimentológica, deve-se considerar ainda que o provável aporte de matéria orgânica proveniente dos restos metabólicos e, especialmente, de ração ofertada aos camarões, pode contribuir para a redução da qualidade do sedimento criando um ambiente desfavorável à sobrevivência dos organismos bentônicos. Lorenzen et al. (1987) afirmam que em ambientes poluídos, onde se tem acúmulo de MO, há decréscimo da 
diversidade desses organismos. Sabe-se que o acúmulo de matéria orgânica pode tornar os sedimentos ácidos e pobres em oxigênio, o que incrementa as vias anaeróbicas de decomposição, aumentando a produção e liberação de compostos tóxicos reduzidos, como amônia, sulfetos, manganês (na forma $\mathrm{Mn}^{+2}$ ), metano e nitrito resultando, por fim, na alteração da estrutura da comunidade bentônica (Jackson et al., 2004).

Finalmente, ficou evidente a ocorrência de uma grande diminuição das densidades dos organismos macrobentônicos na segunda campanha atribuída, portanto, a esta atividade; seja ela devida a uma causa direta (como, por exemplo, a predação, bioturbação, resistência da panagem à dispersão ou colonização etc.) ou indireta (por exemplo, degradação do sedimento). Embora a interferência do cultivo se tenha mostrado de abrangência local e temporária (pois é realizada em apenas 4 meses do ano), o metabolismo bêntico ganha importância ao se considerar que o Saco do Justino é uma enseada rasa e de circulação restrita, diminuindo assim o poder de dispersão dos poluentes e gerando um ambiente mais favorável ao desequilibro ambiental. Portanto, o desenvolvimento desta atividade em escala comercial e em ambientes sensíveis deve ser precedido de uma avaliação criteriosa para se determinar sua viabilidade ambiental e evitar que esta região e o Brasil experimentem situações devastadoras, como as ocorridas no Equador, China, Indonésia e Tailândia.

\section{CONCLUSÕES}

1. Existe clara interferência dos cultivos nos processos de regeneração bêntica de nutrientes, incrementando os fluxos de oxigênio (COS), de nitrogênio (na forma de amônio) e fósforo (fosfato), causando, ainda, uma diminuição da densidade de organismos bentônicos ao final do cultivo.

2. O metabolismo bêntico mostrou-se importante fator regulador dos teores nutrientes e oxigênio na coluna d’água podendo, desta forma, os dados aqui encontrados, serem incorporados a modelos matemáticos preditivos a fim de torná-los mais completos e confiáveis quando utilizados como ferramenta de avaliação de impactos ambientais.

\section{LITERATURA CITADA}

Abreu, P. C. Phytoplankton production and the microbial food web of the Patos Lagoon estuary, southern Brazil. Bremen: Universidade de Bremen, 1992. 100p. Tese Doutorado

Aller, R. C.; Yingst, J. Y. Relationships between microbial distributions and the naerobic decomposition of organic matter in surface sediments of Long Island Sound, USA. Marine Biology, v.56, p.29-42, 1980.

Alongi, D. M., Lindsay, F. T.; Trott, A. Rates and pathways of benthic mineralization in extensive shrimp ponds of the Mekong delta, Vietnam. Aquaculture, v.175, p.269-292, 1999.
Alonso-Rodríguez, R.; Páez-Osuna, F. Nutrients, phytoplankton and harmful algal blooms in shrimp A review with special reference to the situation in the Gulf of California. Aquaculture, v.219, p.317-336, 2003.

Aminot, A.; Chaussepied, M. Manuel des analyses chimiques en milieu marin. Brest: CNEXO, 1983. 395p.

Avnimelech, Y.; Ritvo, G. Shrimp and fish pond soils: Processes and management. Aquaculture, v.220, p.549-567, 2003.

Balls, P. W.; Brockie, N; Dobson, J.; Johnston, W. Dissolved oxygen and nitrification in the upper Forth estuary during Summer 1982-92: Patterns and Trends. Estuarine, Coastal and Shelf Science, v.42, p.117-134, 1996.

Baumgarten, M. G. Z; Rocha, J. M.; Niencheski, L. F. H. Manual de análises em oceanografia química. Rio Grande: FURG 1996, 132p.

Bemvenuti, C. E. Interações biológicas da macrofauna bentônica numa enseada estuarina da Lagoa dos Patos, RS, Brasil. São Paulo: Instituto Oceanográfico/USP, 1992. 206p. Tese Doutorado

Bemvenuti, C. E. O poliqueta Nephtys fluviatilis Monro, 1937 como predador da infauna na comunidade de fundos moles. Atlântica, v.16, p.87-98, 1994.

Bemvenuti, C. E. Fundos não vegetados. IN: Seeliger, U.; Odebrech, C.; Castello, J. P. (eds.). Os ecossistemas costeiro e marinho do extremo sul do Brasil. Rio Grande: Ecoscientia, 1998. p.13-18.

Boyd, C. E. Methods for improving shrimp farming in Central. Management practices for reducing the environmental impacts of shrimp farming. Manágua: UCA University Press, 2001. 292p.

Burford, M. A.; Costanzo, S. D.; Dennison, W. C.; Jackson, C. J.; Jones, A. B.; Mckinnon, A D.; Preston, N. P.; Trott, L. A. A synthesis of dominant ecological processes in intensive shrimp ponds and adjacent coastal environments in NE Australia. Marine Pollution Bulletin, v.46, p.1456-1469, 2003.

Canfield, D. E. Sulfate reduction and oxic respiration in marine sediments: Implications for organic carbon preservation in euxinic environments. Deep-Sea Research, v.36, p.121-138, 1989.

Castello, J. P. La ecologia de los consumidores del estuario da Lagoa dos Patos, Brazil. In: Yanes-Arancibia, A. Fish community ecology in estuaries and coastal lagoons: Towards an ecosystem integration. Mexico: Dr (R) Unam Press, 1985, p.383-406.

Cavalli, R. O., Wasielesky, W., Peixoto, S.; Santos. M. H. S. Estimating the best stocking density for the culture of Farfantepenaeus paulensis in pen enclosures. Salvador: World Aquaculture Society Annual Meeting, 2003. 163p.

Fenchel, W.; Glud, R. N. Small-scale spatial and temporal variability in coastal benthic $\mathrm{O} 2$ dynamics: Effects of fauna activity. Limnology Oceanography, v.49, p.1471-1481, 2004.

Fernandes, E.; Niencheski, L. F. Um modelo de caixas simplificado para o estudo dos processos de transporte na região estuarial da Lagoa dos Patos, RS, Brasil. Atlantida, v.20, p.73-85, 1998.

Forja, J m; Ortega, T.; Delvalls, A.; Gómez-Parra, A. Benthic fluxes of inorganic carbon in shallow coastal ecosystems of the Iberian Península. Marine Chemistry v.85, p.141-156, 2004.

Freitas, U. Regeneração bêntica e qualidade de água em ambientes sob ação de cultivos de camarão (estuário da Lagoa dos Patos). Rio Grande: Fundação Universidade do Rio Grande, 95p. 2006. Dissertação Mestrado 
Glud, R. N.; Rysgaard, S.; Kühl, M. A laboratory study on $\mathrm{O} 2$ dynamics and photosynthesis in ice algal communities: quantification by microsensors, $\mathrm{O} 2$ exchange rates, 14C incubations and a PAM fluorometer. Aquatic Microbial Ecololoy, v.27, p.301-311, 2002.

Hargrave, B. T.; Connolly, G. F. A device to collect supernatant water for measurement of the flux of dissolved compounds across sediment surfaces. Limnology and Oceanography, v.23, p.1005-1010, 1978.

Hargreaves, J. A. Nitrogen biogeochemistry of aquaculture ponds. Aquaculture, v.166, n.3-4, p.181-212, 1998.

Herz, R. Circulação das águas de superfície da Lagoa dos Patos. São Paulo: USP, 1977, 217p. Tese Doutorado.

Hickey, W. C. Quantitative addition of dissolved oxygen to in situ benthic chamber systems by use of catalase and hydrogen peroxide. Applied and Environmental Microbiology, v.49, n.2, p.462-464, 1985.

Hinrichorasen, D. Coastal waters of the world: Trends, threats, and strategies. Washington, DC: Island Press, 1998, 275p.

Jackson, C.; Preston, N.; Thompson, P. J. Intake and discharge nutrient loads at three intensive shrimp farms. Aquaculture Research, v.35, n.11, p.1053-1061, 2004.

Jiménez-Montealegre R.; Verdegem, M. C. J.; Van Dam, A.; Verreth, J. A. J. Conceptualization and validation of a dynamic model for the simulation of nitrogen transformations and fluxes in fish ponds. Ecological Modelling, v.147, p.123-152, 2002.

Jones A. B.; Dennison, W. C.; Preston, N. P. Integrated treatment of shrimp effluent by sedimentation, oyster filtration and macroalgal absorption: A laboratory scale study. Aquaculture, v.193, p.155-178, 2001.

Kantin, R.; Baumgarten, M. G. Z. Observações hidrográficas nos estuário da Lagoa dos Patos: Distribuição e flutuações dos sais nutrientes. Atlântica, v.5, n.1, p.76-92, 1982.

Lacerda L d.; Vaisman A. G.; Maia L. P.; Ramos e Silva C. A.; Cunha, E. M. S. Relative importance of nitrogen and phosphorus emissions from shrimp farming and other anthropogenic sources for six estuaries along the NE Brazilian coast. Aquaculture, v.253, p.433-446, 2006.

Lin, C. K.; Nash, G. L. Asian shrimp news, collected columns, 1989 - 1995. Bangkok: Asian Shrimp Culture Council, 1996, 312p.

Lorenzen, S.; Prein, M.; Valentin, C. Mass aggregations of the free-living marine nematode Pontonema vulgare Oncholaimidae in organially polluted fjords. Marine Ecology Progress Series, v.37, p.27-34, 1987.

Machado, E. C. Desoxigenação e regeneração de nutrientes pelo sedimento na Lagoa de Guarapina: UFF, 1989. 104p. Dissertação Mestrado
Nazário, M.; Machado, E.; Brandini, B.; Mizerkowski, B. D. Consumo de oxigênio pelo sedimento e fluxos de nutrientes na interface água-sedimento em uma área de cultivo de ostras (Crassostrea sp.) na baía de Guaratuba, Paraná, Brasil. Congresso Brasileiro de Oceanografia, 2, 2005, Vitória. Anais.... Vitória: Associação Brasileira de Oceanografia, 2005, CD Rom

Pereira Filho, J.; Oliveira, U. C.; Manzoni, G. C. O uso de Bell Jar na avaliação do metabolismo bentônico na armação do Itapocoroy: Resultados preliminares. Notas técnicas FACIMAR, v.2, p.81-92, 1998.

Poersch, L. H. S. Aqüacultura no estuário da Lagoa dos Patos e sua influência sobre o meio ambiente. Rio Grande: FURG, 2004, 146p. Tese Doutorado

Riise, J. C.; Roos, N. Benthic metabolism and the effects of bioturbation in a fertilized polyculture fish pond in northeast Thailand. Aquaculture, v.150, p.45-62, 1997.

Schenau, S. J.; de Lange, G. J. Phosphorus regeneration vs. burial in sediments of the Arabian Sea. Marine Chemistry, v.75, p.201-217, 2001.

Sigmon, D. E.; Cahoon, L. B. Comparative effects of benthic microalgae and phytoplankton on dissolved silica fluxes. Aquatic Microbial Ecology, v.13, p.275-284, 1997.

Soares, R.; Peixoto, S.; Bemvenuti, C.; Wasielesky, W.; D’incao, F.; Murcia, N. E.; Suita, S. Composition and abundance of invertebrate benthic fauna in Farfantepenaus paulensis culture pens (Patos Lagoon estuary, southern Brazil). Aquaculture, v.239, p.199-215, 2004.

Solorzano, L. Determination of ammonia in natural waters by the phenolhypochlorite method. Limnology and Oceanography, v.14, n.5, p.799-801, 1969.

Strickland, J. L. H.; Parsons, T. R. A practical handbook of seawater analysis. Othawa: Fisheries Research Board of Canada, 1972. 310p. Bulletim 167

Thamdrup, B.; Canfield, D. E. Benthic respiration in aquatic sediments. In: Sala, O.; Mooney, H.; Jackson, R.; Howarth, R. (ed.). Methods in ecosystem science. New York: Springer, 2000, p.86-103.

Trott, L. A.; Mckinnon, A d.; Alongi, D m.; Davidson, A.; Burford, M. A. Carbon and nitrogen processes in a mangrove creek receiving shrimp farm effluent. Estuarine, Coastal and Shelf Science, v.59, p.197-207, 2004.

Ward, B. B. Nitrification and the marine nitrogen cycle. In: D. Kirchman, (ed) New York: Microbial Ecology, Wiley-Liss, 2000, p.427-454.

Warnken, K. W.; Gill, G. A.; Lehman, R d.; Dellapenna, T m.; Allison, M. A. The effects of shrimp trawling on sediment oxygen demand and the release of trace metals and nutrients from estuarine sediments. Estuarine Coastal and Shelf Science, v.57, p.25-42, 2002.

Zarzur, Z. Consumo de oxigênio pelo sedimento e fluxos de regeneração bêntica de nutrientes nas áreas rasas do estuário da Lagoa dos Patos, RS. Rio Grande: FURG, 2001, 147p. Dissertação Mestrado 